\title{
RELATIONSHIP BETWEEN TRAINING LOAD INDICATORS AND TRAINING PERIODIZATION DURING PRESEASON IN ELITE FOOTBALL GOALKEEPERS
}

original paper

(1) University School of Physical Education in Wroclaw

DOI: https://doi.org/10.5114/hm.2018.83217

\section{LUIS ESTEVES, PEDRO SANTOS, CARLOS LAGO-PENAS}

University of Vigo, Vigo, Spain

\begin{abstract}
Purpose. To examine the training load of goalkeepers in professional football.

Methods. Overall, 74 individual preseason training sessions of 3 clubs' goalkeepers were analysed with the use of the Global Positioning System. The total of 12 physical parameters (total distance, high speed running, high metabolic load distance, high metabolic load efforts, high metabolic load time, sprint, maximum speed, acceleration, deceleration, impacts, dynamic stress load, and step balances) were compared depending on the perceived exertion by the goalkeepers' coach (low, medium, or high training load). The ANOVA with post-hoc least significant difference, Bonferroni and Gabriel tests, or alternatively Kruskal-Wallis and Mann-Whitney tests were used $(p<0.05)$. The effect size and Pearson coefficients of variation were also calculated.
\end{abstract}

Results. The dynamic stress load, impacts, acceleration, deceleration, high metabolic load time, and total distance appear to have superior values assigned to the session type with higher workout load design. Unexpectedly, this trend is reverse for maximum speed, sprint, and high speed running load comparisons, although the differences attributed to sprint seem to have no practical value.

Conclusions. Training monitoring is needed to ensure that the load applied matches the intentions of the coach's prescription and meets the purposes of accurate periodization. This information can be used to better understand goalkeepers' training load during preseason.

Key words: performance analysis, training monitoring, physical outcome, GPS, periodization

\section{Introduction}

Performance analysis in football aims to increase the knowledge of the players' match performance, which refers also to goalkeepers (GKs). However, there is a lack of studies concerning the match and training activity of GKs. For example, some align with the technical performance domain of GKs, considering situational variables [1], intervention characteristics in parallel with features of the opponent [2] or propose a model focused on game actions [3]. The GK performance was also evaluated with reference to saves-toshots ratio (shot path, trajectory, and style) [4]. Aspects related to the penalty kick were also targeted [5]. Another line of research profiles anthropometric (e.g. anthropometry, somatotype, and body composition) and fitness (e.g. endurance, sprint, and jump tests) characteristics of players, depending on their position (GKs included) [6]. A review of a series of studies concerning this issue can be found elsewhere [7].

Different instruments have been used to monitor the external load of football players, such as video tracking systems [8] or the Global Positioning System (GPS) [9]. Comparisons of the results between various match analysis systems must be performed with caution [10]. The physical performance of players at different positions and competitive standards is properly documented $[11,12]$ but the same cannot be stated about GKs since most studies exclude these team members from analysis. Di Salvo et al. [13] and Malone et al. [14] are exceptions. The former paper analyses the distances covered by GKs during a match at different

Correspondence address: Luís Esteves, Faculty of Education and Sport Sciences, University of Vigo,

Campus de A Xunqueira s/n. CP: 36005, Pontevedra, Spain, e-mail: lmmesteves@gmail.com

Received: October 25, 2018

Accepted for publication: January 29, 2019

Citation: Esteves L, Santos P, Lago-Penas C. Relationship between training load indicators and training periodization during preseason in elite football goalkeepers. Hum Mov. 2018;19(5)special/issue:89-97; doi: https://doi.org/10.5114/hm.2018.83217. 
velocities. A limitation is that high intensity actions such as clearing, controlling, and saving were not considered [7]. The latter paper is an exploratory case study that attempted to quantify the load-response relationship during a competitive season. However, this study focuses only on a single GK, and the generalization of the findings becomes an issue. There is a poverty of studies that examine the physical external load of GKs, either in match or in training. This constitutes an important limitation. Indeed, football GKs are required to perform strenuous actions during training sessions and actual games. Therefore, obtaining relevant information about their physical characteristics and physiological attributes supports planning training programs for them, for different phases of the season [7].

The effective planning and organization of training are crucial to the effective delivery of training stimuli [15]. Training monitoring is critical to the process of quantifying training periodization plans [16] and for improvements in the preparation, training, and recovery aspects [17]. Thus, continuous monitoring of all training sessions and matches is recommended for future research in order to provide a clear insight into the performance workload [18]. Given the validity and reliability of the data collected by GPS [19, 20], its use to perform time-motion analysis in football has become popular, and several examples can be found in the literature [e.g. 9]. There are a large number of variables that can be measured by GPS devices [21]. The possible performance indicators applied to evaluate physical performance were extended, which allowed to go beyond the measure of the running distances of players at different speed thresholds, especially owing to the complementary use of accelerometers integrated in GPS units [22]. For instance, Gaudino et al. [23] include the dynamic-stress load, and Bauer et al. [21] point at the player load. This information allows more accurate training plans.

The aim of this study was to determine the characteristics of GKs external load during a preseason training within a professional club setting. It was hypothesized that the GKs of the sampled team altered the type of training load in accordance with the purposes of training periodization. Differences in the existing physical demands should be superior in the high load training sessions defined by the coach as compared with moderate or low load training sessions.

\section{Material and methods}

\section{Participants and data collection procedures}

Training load data were collected with the use of GPS during 28 preseason trainings of a 2015-2016 Portuguese Premier League team, which also participated in European competitions. The preseason lasted from June $27^{\text {th }}$ to July $22^{\text {nd }}$, including 21 days of training, with 7 two-day trainings. It was a short preseason owing to a prequalification UEFA Europa League match. A 4-week training took place before the first competitive microcycle, whereas the first official game appeared in the $5^{\text {th }}$ week of training. The first microcycle for the competitive period was not included as the study was focused on preseason. Therefore, 74 individual preseason training sessions (27 with low training load, 22 with medium training load, and 25 with high training load, as classified by the GK coach according to the criteria defined in Table 1) of 3 clubs' GKs (age: $26.3 \pm 5.5$ years; body weight: $88.3 \pm 3.2 \mathrm{~kg}$; height: $1.90 \pm 4.9 \mathrm{~m}$ ) were examined. Because of an injury, one of the GKs only took part in the first 18 trainings. The remaining GKs participated in all the preseason sessions. There were 2 missing values in the variable of high metabolic load time for $1 \mathrm{GK}$ ( 1 concerning the low training load, 1 for the medium training load). The trainings took place on a natural grass field. All GKs were familiar with the training protocols prior to the investigation.

The GKs physical activity during each training session was monitored with a portable GPS unit (Viper pod, version 1.2, STATSports, Northern Ireland). The device provides position velocity and distance data at $10 \mathrm{~Hz}$. Each Viper pod contains 4 processors, 3-dimensional accelerometers, a 3-dimensional gyroscope, a 3-dimensional digital compass, a long range radio, and a heart rate receiver. These components log data at a rate of up to $100 \mathrm{~Hz}$ and stream data at over $50 \mathrm{~Hz}$.

GPS devices have been recognized as valid and reliable tools for measuring total distance, high speed distance, sprints, and other. These are among the data that the new feature has access to [24, 25].

\section{Variables}

GPS data were downloaded on a daily basis and included 12 physical parameters: total distance (TD), high speed running (HSR), high metabolic load distance (HMLd), high metabolic load efforts (HMLe), high metabolic load time (HMLt), sprint (Sp), maximum speed (MaxS), acceleration (Ace), deceleration 
(Des), impacts (Imp), dynamic stress load (DSL), and step balances (SB). The data were analysed in relation to 3 training load variables (low, medium, or high training load) that were sorted by the GK coach in accordance with the planned exertion definition. The definitions of low, medium, and high training load were developed with the GK coach and validated by an academic researcher and a strength and conditioning coach. Operational definitions of these variables are displayed in Tables 1 and 2 .

\section{Statistical analysis}

The one-way analysis of variance (ANOVA) with post-hoc least significant difference, Bonferroni, and Gabriel tests or, alternatively, when the assumptions

Table 1. Characteristics of the goalkeepers' training loads (independent variable of the study)

Low training load As in all training sessions planned to have an impact that is too low in terms of external physical loads. The parameters associated with recovery are enhanced (e.g., relationship between time of action and rest to promote fatigue prevention and the type of exercises selected)

Medium training load As in all training sessions planned to have an impact that is not considered too low or too high in terms of external physical loads. The parameters associated with high load are enhanced (e.g., relationship between time of action and rest and the type of exercises selected)

High training load As in all training sessions planned to have an impact that is excessively high in terms of external physical loads. The importance of high-intensity training bouts is promoted. The parameters associated with very high load are enhanced (e.g., relationship between time of action and rest and the type of exercises selected)

Table 2. Characteristics of the physical parameters used in the study

\begin{tabular}{|c|c|}
\hline Parameter & Description \\
\hline Total distance & - Total distance during the whole training session (measured in meters) \\
\hline High speed running & $\begin{array}{l}\text { - Distance covered in zones } 5 \text { and } 6 \text { (measured in meters) } \\
\text { - Zone } 5(>5.5 \mathrm{~m} / \mathrm{s}) \text {, zone } 6(>7 \mathrm{~m} / \mathrm{s})\end{array}$ \\
\hline High metabolic load distance & $\begin{array}{l}\text { - Includes both high speed running distance and distance covered while accelerating } \\
\text { or decelerating above } 2 \mathrm{~m} / \mathrm{s}^{2} \text { (measured in meters) }\end{array}$ \\
\hline High metabolic load efforts & $\begin{array}{l}\text { - Shows the number of times the high metabolic load distance occurs throughout } \\
\text { the training session }\end{array}$ \\
\hline High metabolic load time & - Shows the time spent covering high metabolic load distance \\
\hline Sprint & $\begin{array}{l}\text { - A number rather than a distance; number of sprint entries (default }>5.5 \mathrm{~m} / \mathrm{s} \text {, zone } 5 \text { ). } \\
\text { Speed must be maintained for }>1 \mathrm{~s} \text { for sprint to be registered }\end{array}$ \\
\hline Maximum speed & - Measure of total exertion based on 'time at speed' (m/s) \\
\hline Acceleration & $\begin{array}{l}\text { - Gives the total number of accelerations based off zone preference selection } \\
\text { - For an acceleration to be registered, an increase in speed of at least } 0.5 \mathrm{~m} / \mathrm{s}^{2} \\
\text { must occur for at least } 0.5 \mathrm{~s}\end{array}$ \\
\hline Deceleration & $\begin{array}{l}\text { - Gives the total number of decelerations based off zone preference selection } \\
\text { - Decelerations are measured in exactly the same way as accelerations: a decrease } \\
\text { in speed of at least } 0.5 \mathrm{~m} / \mathrm{s}^{2} \text { must occur for at least } 0.5 \mathrm{~s}\end{array}$ \\
\hline Impacts & - Shows the total number of impacts \\
\hline Dynamic stress load & $\begin{array}{l}\text { - Total of the weighed impacts. In a non-collision sport such as football, the majority } \\
\text { of these impacts are dominated by running steps } \\
\text { - Therefore the dynamic stress load for a session represents the loading effect } \\
\text { on the body }\end{array}$ \\
\hline Step balances & $\begin{array}{l}\text { - Involves the use of an accelerometer and determines the average peak impact } \\
\text { with each step for the left and the right foot }\end{array}$ \\
\hline
\end{tabular}


of parametric tests were violated, Kruskal-Wallis and Mann-Whitney tests were used to examine differences across the training loads. The significance level was set at 5\%. Effect size $(E S)$ was calculated to determine the practical meaningfulness of the difference. The threshold values for Cohen's $E S$ were as follows: $>0.2$ (small), $>0.6$ (medium), and > 1.2 (large) [26]. To examine the training-to-training variability from each training load category of the GKs, the Pearson coefficients of variation $(\mathrm{CV})$ were calculated. To express the percentage, the value was multiplied by 100 . Data analysis was processed with the use of the SPSS for Windows software, version 22 (IBM Corp., Armonk, NY, USA).

\section{Ethical approval}

The research related to human use has been complied with all the relevant national regulations and institutional policies, has followed the tenets of the Declaration of Helsinki, and has been approved by the Ethics Committee of the Faculty of Education and Sport Sciences, University of Vigo, Spain.

\section{Informed consent}

Informed consent has been obtained from all individuals included in this study.

\section{Results}

Table 3 includes the descriptive statistics for the physical performance indicators as a function of the training load defined by the coach.

Considering the training load, significant differences were found for DSL, Des, Imp, HMLt $(p<0.01$, with parametric tests), and HSR, Ace, MaxS, Sp ( $p<$ $0.01)$ and TD $(p<0.05)$ (with non-parametric tests). No significant differences were found for HMLd, HMLe, or SB $(p \geq 0.05)$.

\section{Comparison of low vs. medium training load}

$\operatorname{HSR}(p<0.01, E S=0.81), \operatorname{MaxS}(p<0.01, E S=1.08)$, and $\operatorname{Sp}(p<0.01, E S=1.12)$ were inferior for the medium training load, with $14.8 \mathrm{~m}, 1.2 \mathrm{~s}$, and 1 sprint less in median, respectively. $E S$ values were classified as medium. CVs equalled, respectively, 167.4, 16.9, and 113.3 percent for the low training load and 185.7, 12.7, and 500 percent for the medium training load.

$\operatorname{DSL}(p<0.01, E S=1.65)$, Ace $(p<0.05, E S=0.81)$, Des $(p<0.01, E S=1.12)$, and $\operatorname{Imp}(p<0.01, E S=2.08)$ had superior values for the medium training load, with 47.3 mean load, 10.5 accelerations (in median), 13.5 decelerations, and 29.2 impacts more in mean terms, respectively. $E S$ values were classified as medium (e.g. Ace, Des) and large (e.g. DSL, Imp). CVs equalled, respectively, 48.0, 52.5, 44.1, and 58.2 percent for the low training load and 24.4, 44.0, 39.4, and 25.9 percent for the high training load.

\section{Comparison of low vs. high training load}

HSR $(p<0.01, E S=0.75), \operatorname{MaxS}(p<0.01, E S=$ $1.13)$, and $\operatorname{Sp}(p<0.05, E S=0.72)$ were inferior for the high training load, with $14.8 \mathrm{~m}, 1.1 \mathrm{~s}$, and 1 sprint less in median, respectively. $E S$ values were classified as medium. $C V$ s equalled, respectively, $167.4,16.9$, and 113.3 percent for the low training load and 183.9, 10.9, and 200 percent for the high training load.

DSL $(p<0.01, E S=3.63)$, Ace $(p<0.01, E S=1.39)$, Des $(p<0.01, E S=1.89), \operatorname{Imp}(p<0.01, E S=4.00)$, and HMLt $(p<0.01, E S=1.13)$ had superior values for the high training load, with 104.6 mean load, 16.0 accelerations (in median), 23.4 decelerations, 57.0 impacts, and $17 \mathrm{~s}$ more on average, respectively. ES values were classified as large. $C V$ s equalled, respectively, 48.0, 52.5, 44.1, 58.2, and 27.8 percent for the low training load and 16.2, 40.1, 32.2, 17.6, and 21.1 percent for the high training load.

\section{Comparison of medium vs. high training load}

$\mathrm{TD}(p<0.05, E S=0.97), \mathrm{DSL}(p<0.01, E S=2.12)$, Des $(p<0.05, E S=0.69)$, $\operatorname{Imp}(p<0.01, E S=2.04)$, and HMLt $(p<0.05, E S=0.76)$ were superior for the high training load, with $676.5 \mathrm{~m}, 57.1$ load, 9.9 decelerations, 27.8 impacts, and $13 \mathrm{~s}$ more, respectively. $E S$ values were classified as medium (e.g. TD, Des, HMLt) and large (e.g. DSL, Imp). CVs equalled, respectively, $18.3,24.4,39.4,25.9$, and 32.8 percent for the medium training load and 12.6, 16.1, 32.2, 16.3, and 21.1 for the high training load.

With reference to the comparisons established, Figure 1 displays the significant differences within the training load comparisons. The $E S$ is also qualified.

\section{Discussion}

The aim of the current study was to carry out the performance assessment of GK physical load during preseason training within a professional club setting. Training loads were adjusted at various times during the training cycle [27]; as hypothesized, the type of training altered in accordance with low, medium, or high load. These conclusions are in line with the scientific literature suggesting that appropriate periodization 
Table 3. Descriptive statistics (mean $[M]$ and standard deviation $[S D]$, Pearson coefficients of variation $[C V]$ expressed as percentage, Cohen's effect sizes [ES], and median $[M d n]$ and interquartile range [IQR], but only when parametric tests assumptions were violated) of physical performance indicators as a function of the training load defined by the coach

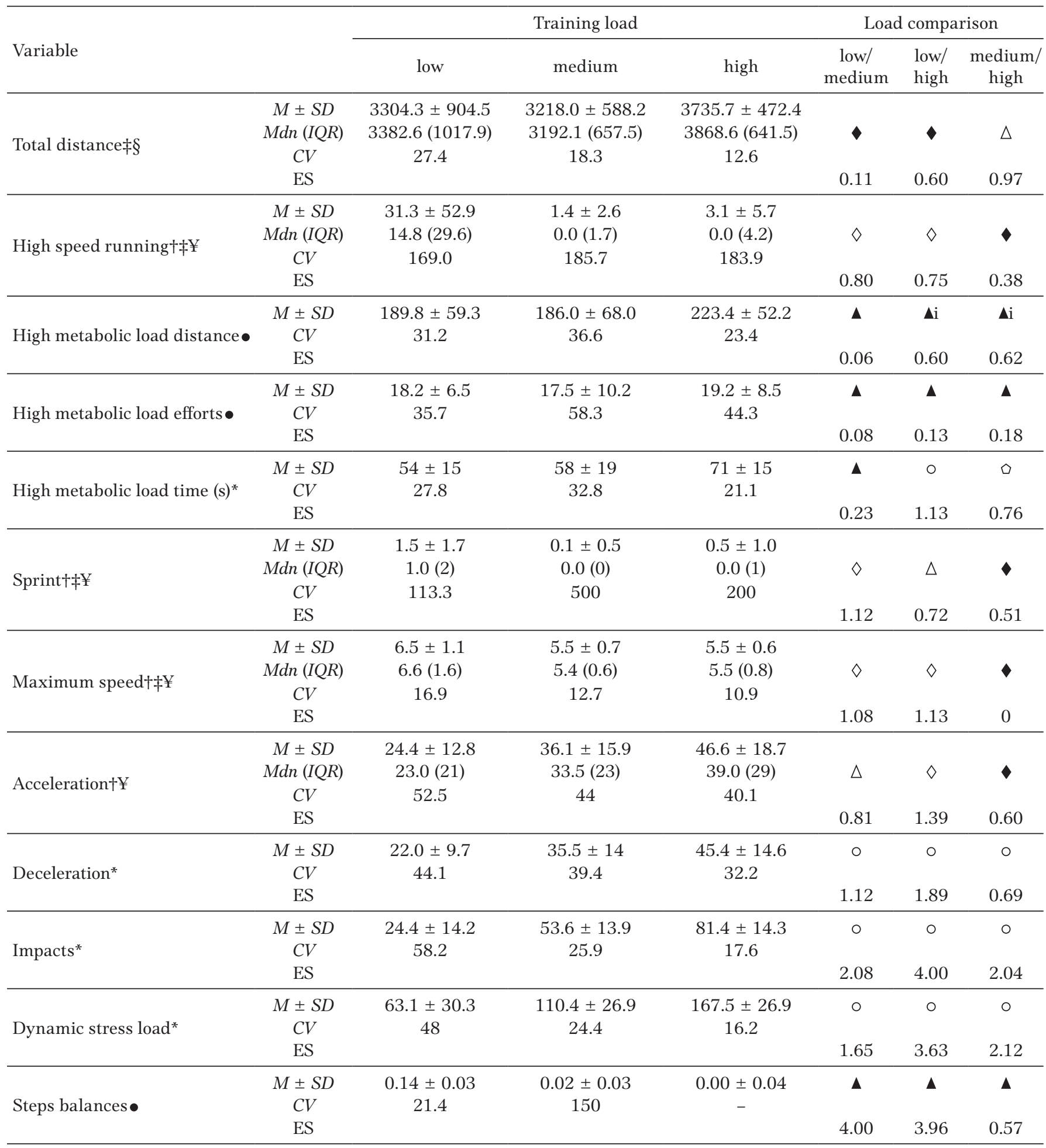

† The normality assumption was rejected (Shapiro-Wilk test, $p<0.05$ )

$\ddagger$ The equality of variances was not assumed (Levene’s test, $p<0.05$ )

ANOVA (homogeneity of variance assumed): ${ }^{*} p<0.01, \bullet p \geq 0.5$

post-hoc tests (least significant difference, Bonferroni, Gabriel): $\mathbf{\Delta} p \geq 0.5, \diamond p<0.5, \circ p<0.1$,

$\Delta$ l least significant difference test, $p<0.5$; Bonferroni, Gabriel test, $p \geq 0.5$

When parametric tests assumptions were violated: Kruskal-Wallis test: $¥ p<0.01, \S p<0.05$;

Mann-Whitney test: $\diamond p<0.01, \Delta p<0.05, \diamond p \geq 0.05$ 


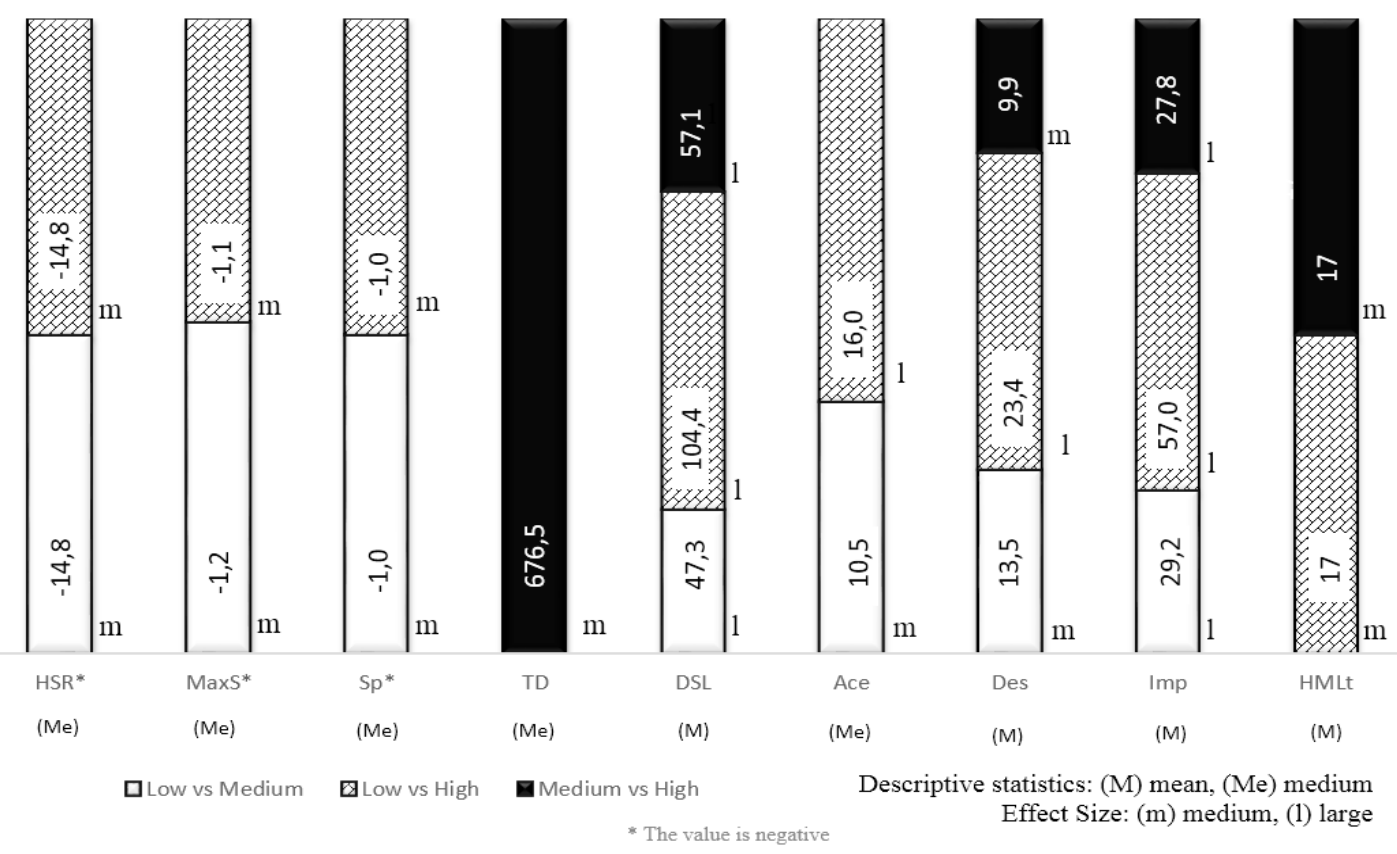

Figure 1. Significant differences for the training load comparisons

strategies are essential to high performance [15, 28].

Appropriate monitoring of the training load can provide crucial information for the improvement of performance while preventing injuries [18, 22, 27]. The GK actions typically involve explosive, shortduration movements, such us diving, catching, and accelerating/decelerating sharply [7], which are often required in repeated successions [14]. Out of the performance indicators examined, DSL, Des, and Imp appear to be the most significant to differentiate the type of training load of a GK. They consistently differentiate the studied training categories, with the values of the high load training more elevated than in the medium load and these, in turn, higher than in the low load. Other variables seem to be significant to assess the GK's load, with Ace differentiating the low load from the medium or high load, HMLt differentiating the high load from the low or medium load, and TD differentiating only the high from the medium load. It must be recognized that for all these variables the $E S$ values were classified as medium to large. The $C V$ s were inferior for the high load sessions. A general pattern is evidenced in these significant comparisons, resulting in higher values in the GK external load corresponding to the session type with higher workout load design defined by the coach. For instance, a GK covers more than $676.5 \mathrm{~m}$ during a high load training, which is more than in medium load sessions (totalizing $3192 \mathrm{~m}$ ). Interestingly, however, there are no differences between low and medium or low and high load training. This may be due to the position- specific practices of the GKs, who often train in small groups using position-specific training drills delivered by the GK coach, with some involvement in outfield player drills (e.g., tactical and small-sided game drills) [14]. Although the TD variable should be taken into account when assessing the GK physical performance, care should be taken as it does not involve all the comparison scenarios. From this point of view, TD is not a major variable to discern in the GK training process. It is an indicator of external loading more commonly associated with the volume of training rather than training intensity [29]. It is known that GK-specific training is characterized by short-burst movements, maintained for more repetitions with lower pauses, under multidirectional and physically demanding tasks. The TD values found during preseason in this study are close to those observed by Malone et al. [14] with a single GK across a season and range between 2553 and $3742 \mathrm{~m}$. This distinguishes GKs from the outfield players, with lower TD values in matches, e.g. $5611 \mathrm{~m}$ for a GK [13] vs. 10,714 $\mathrm{m}$ for an outfield player [30], and during training sessions, e.g. the mean of $6182 \mathrm{~m}$ for an in-season week 7 [24] or $6871 \mathrm{~m}$ in a high intensity session [29].

Significant differences were also found for MaxS, Sp, and HSR between the low and medium load and between the low and high load. Superior values appeared in the low load training category, which was not expected. To explain this, it is necessary to take into account the nature of the actions performed by GKs. From a practical point of view, a specific work 
calling for typical GK actions may be more related to medium load or high load sessions. It is possible that the low load sessions are usually characterized by reaction speed actions and linear sprint drills alongside with outfield players, which may explain these higher values. It was found that when the high speed distances covered between positions are compared, GKs appear to cover only a minor part of outfield players distances (ca. 17\%); this is considered most likely due to the restriction in space for the majority of the GK-specific training drills, which limits the ability to reach higher speed thresholds [14]. Notwithstanding the small differences in the median of MaxS, the values are sorted with different thresholds, with the low load and the high load reaching 'high-speed running,' and the median load attaining the superior edge of the 'running' category [30-32]. The difference found in Sp appears to have no practical value. Furthermore, despite the medium $E S$ observed, the $C V$ values were very high. Seeing that speed must be maintained for $>1 \mathrm{~s}$ to register a sprint, the restricted number of sprint entries noted with this variable (either average or median) may suggest that a GK performs speeds > $5.5 \mathrm{~m} / \mathrm{s}$ for shorter periods. It would be appropriate to consider a record over a 0.5 -second time interval, as done elsewhere $[8,31,33]$. Interestingly, it may be stated that during the low load training, a GK covers a few meters more at HSR. One must be aware that the $C V$ expresses high values. The HSR recorded encompasses 2 thresholds for locomotor categories [30-32], representing a very high intensity domain [34], which may involve a higher injury risk [18]. Future research needs to ascertain the type of exercises used during these trainings and its application context to truly understand the higher MaxS and HSR values in low load training sessions compared with the medium or high load trainings.

Usually, there are only 3 GKs in each professional football squad. This narrows the sample within a club, which constitutes a limitation of this research. It would also be important to monitor the heart rate and the session rating of perceived exertion during the training sessions to include evaluation of the internal load $[16,18,27]$. Further work is required to examine the magnitude of periodization practices, in accordance with the quantification of GK training load. It may be argued that the terminology used for the intensity scale is somewhat vague. Future studies can outline a better characterization of the GK training load (low, medium, and high), based on the information collected in this study about the variables sensible to the GK load periodization and on the opinion of a group of experts in goalkeeping coaching. Also, it is important to ascertain the effect of the duration (e.g. over TD) and type of training (e.g. position-specific, integrated with outfield players, or general training). Practical applications of training load monitoring should be addressed to coaches and athletes. The process of continuous monitoring could lead to a better insight into the actual training load and potentially result in changes in the training prescription in subsequent sessions (i.e. load increase or decrease) [18]. There is a need to examine load-performance relationships $[24,27]$ and extend the analysis to the demands of GKs during competition. This should be investigated with reference to situational variables $[1,35,36]$.

\section{Conclusions}

The presented study confirms the existence of a differentiated typology of load design in preseason GK training sessions. The high, medium, or low load classification provided by the coach was validated. The DSL, Des, and Imp appear to be the most relevant to differentiate the type of the GK training load, followed by Ace, HMLt, MaxS, Sp, and HSR, and, lastly, by TD, with significant differences established within the comparisons. In DSL, Imp, Ace, Des, HMLt, and TD, superior values emerge assigned to the session type with higher workout load design. This trend is reverse for MaxS, Sp, and HSR load comparisons. One of the virtues of training load monitoring is to verify that the player load performance corresponds to the intentions of the planning and periodization.

\section{Disclosure statement}

No author has any financial interest or received any financial benefit from this research.

\section{Conflict of interest}

The authors state no conflict of interest.

\section{References}

1. Liu H, Gómez MA, Lago-Peñas CL. Match performance profiles of goalkeepers of elite football teams. Int J Sports Sci Coach. 2015;10(4):669-682; doi: 10.1260/1747-9541. 10.4.669.

2. De Baranda PS, Ortega E, Palao JM. Analysis of goalkeepers' defence in the World Cup in Korea and Japan in 2002. Eur J Sport Sci. 2008;8(3):127-134; doi: 10.1080/17461390801919045.

3. Oberstone J. Comparing English Premier League goalkeepers: identifying the pitch actions that differentiate the best from the rest. J Quant Anal Sports. 2010;6(1): 9; doi: 10.2202/1559-0410.1221. 
L. Esteves, P. Santos, C. Lago-Penas, Training load and periodization in goalkeepers

4. Gelade G. Evaluating the ability of goalkeepers in English Premier League football. J Quant Anal Sports. 2014;10(2):279-286; doi: 10.1515/jqas-2014-0004.

5. Noël B, van der Kamp J, Memmert D. Implicit goalkeeper influences on goal side selection in representative penaltykickingtasks.PLoSOne.2015;10(8):e0135423; doi: 10.1371/journal.pone.0135423.

6. Nikolaidis P, Ziv G, Arnon M, Lidor L. Physical and physiological attributes of soccer goalkeepers - should we rely only on means and standard deviations? J Hum Sport Exerc. 2015;10(2):602-614; doi: 10.14198/jhse. 2015.102.07.

7. Ziv G, Lidor R. Physical characteristics, physiological attributes, and on-field performances of soccer goalkeepers. Int J Sports Physiol Perform. 2011;6(4):509524; doi: 10.1123/ijspp.6.4.509.

8. Gregson W, Drust B, Atkinson G, Di Salvo V. Match-tomatch variability of high-speed activities in Premier League soccer. Int J Sports Med. 2010;31(4):237-242; doi: 10.1055/s-0030-1247546.

9. Anderson L, Orme P, Di Michele R, Close GL, Morgans R, Drust B, et al. Quantification of training load during one-, two- and three-game week schedules in professional soccer players from the English Premier League: implications for carbohydrate periodisation. J Sports Sci. 2016;34(13):1250-1259; doi: 10.1080/ 02640414.2015.1106574.

10. Buchheit M, Allen A, Poon T, Modonutti M, Gregson W, Di Salvo W. Integrating different tracking systems in football: multiple camera semi-automatic system, local position measurement and GPS technologies. J Sports Sci. 2014;32(20):1844-1857; doi: 10.1080/02640414. 2014.942687.

11. Bradley PS, Carling C, Gomez Diaz A, Hood P, Barnes C, Ade J, et al. Match performance and physical capacity of players in the top three competitive standards of English professional soccer. Hum Mov Sci. 2013;32(4): 808-821; doi: 10.1016/j.humov.2013.06.002.

12. Mohr M, Krustrup P, Bangsbo J. Match performance of high-standard soccer players with special reference to development offatigue. J Sports Sci. 2003;21(7):519528; doi: 10.1080/0264041031000071182.

13. Di Salvo V, Benito PJ, Calderón FJ, Di Salvo M, Pigozzi F. Activity profile of elite goalkeepers during football match-play. J Sports Med Phys Fitness. 2008;48(4): 443-446.

14. Malone JJ, Jaspers A, Helsen W, Merks B, Frencken WGP, Brink MS. Seasonal training load and wellness monitoring in a professional soccer goalkeeper. Int J Sports Physiol Perform. 2018;13(5):672-675; doi: 10.1123/ ijspp.2017-0472.

15. Morgans R, Orme P, Anderson L, Drust B. Principles and practices of training for soccer. J Sport Health Sci. 2014;3(4):251-257; doi: 10.1016/j.jshs.2014.07.002.

16. Foster C, Florhaug JA, Franklin J, Gottschall L, Hrovatin $\mathrm{LA}$, Parker S, et al. A new approach to monitoring
exercisetraining.J Strength Cond Res. 2001;15(1):119_ 115; doi: 10.1519/00124278-200102000-00019.

17. Dellaserra CL, Gao Y, Ransdell L. Use of integrated technology in team sports: a review of opportunities, challenges, and future directions for athletes. J Strength Cond Res. 2014;28(2):556-573; doi: 10.1519/JSC.0b013e3182 a952fb.

18. Jaspers A, Brink MS, Probst SG, Frencken WG, Helsen WF. Relationships between training load indicators and training outcomes in professional soccer. Sports Med. 2017;47(3):533-544; doi: 10.1007/s40279-0160591-0.

19. Aughey RJ. Applications of GPS technologies to field sports. Int J Sports Physiol Perform. 2011;6(3):295-310; doi: 10.1123/ijspp.6.3.295.

20. Varley MC, Fairweather IH, Aughey RJ. Validity and reliability of GPS for measuring instantaneous velocity during acceleration, deceleration, and constant motion. J Sports Sci. 2012;30(2):121-127; doi: 10.1080/0264 0414.2011.627941.

21. Bauer AM, Young W, Fahrner B, Harvey J. GPS variables most related to match performance in an elite Australian football team. Int J Perform Anal Sport. 2015;15(1):187-202; doi:10.1080/24748668.2015.118 68786.

22. Vanrenterghem J, Nedergaard NJ, Robinson MA, Drust B. Training load monitoring in team sports: a novel framework separating physiological and biomechanical load-adaptation pathways. Sports Med. 2017;47(11): 2135-2142; doi: 10.1007/s40279-017-0714-2.

23. Gaudino P, Iaia FM, Strudwick AJ, Hawkins RD, Alberti G, Atkinson G, et al. Factors influencing perception of effort (session rating of perceived exertion) during elite soccer training. Int J Sports Physiol Perform. 2015;10(7):860-864; doi: 10.1123/ijspp.2014-0518.

24. Malone JJ, Di Michele R, Morgans R, Burgess D, Morton JP, Drust B. Seasonal training-load quantification in elite English Premier League soccer players. Int J Sports Physiol Perform. 2015;10(4):489-497; doi: 10.1123/ ijspp.2014-0352.

25. Reilly B, Akubat I, Lyons M, Collins DK. Match-play demands of elite youth Gaelic football using global positioning system tracking. J Strength Cond Res. 2015; 29(4):989-996; doi: 10.1519/JSC.0000000000000714.

26. Cohen J. Statistical power analysis for the behavioral sciences. New York: Lawrence Erlbaum Associates; 1988.

27. Halson SL. Monitoring training load to understand fatigue in athletes. Sports Med. 2014;44(Suppl 2):S139S147; doi: 10.1007/s40279-014-0253-z.

28. Bompa T, Buzzichelli C. Periodization training for sports. Champaign: Human Kinetics; 2015.

29. Owen AL, Wong del P, Dunlop G, Groussard C, Kebsi W, Dellal A, et al. High-intensity training and salivary immunoglobulin A responses in professional top-level soccer players: effect of training intensity. J Strength Cond Res. 2016;30(9):2460-2469; doi: 10.1519/JSC.00000 00000000380 . 
30. Bradley PS, Sheldon W, Wooster B, Olsen O, Boanas P, Krustrup P. High-intensity running in English FA Premier League soccer matches. J Sports Sci. 2009; 27(2):159-168; doi: 10.1080/02640410802512775.

31. Di Salvo V, Gregson W, Atkinsin G, Tordoff P, Drust B. Analysis of high intensity activity in Premier League soccer. Int J Sports Med. 2009;30(3):205-212; doi: 10.1055/s-0028-1105950.

32. Rampinini E, Coutts AJ, Castagna C, Sassi R, Impellizzeri FM. Variation in top level soccer match performance. Int J Sports Med. 2007;28(12):1018-1024; doi: 10.1055/s-2007-965158.

33. Carling C, Bradley P, McCall A, Dupont G. Match-tomatch variability in high-speed running activity in a professional soccer team. J Sports Sci. 2016;34(24): 2215-2223; doi: 10.1080/02640414.2016.1176228.

34. Carling C. Influence of opposition team formation on physical and skill-related performance in a professional soccer team. Eur J Sport Sci. 2011;11(3):155-164; doi: 10.1080/17461391.2010.499972.

35. Castellano J, Blanco-Villaseñor A, Álvarez D. Contextual variables and time-motion analysis in soccer. Int $\mathrm{J}$ SportsMed.2011;32(6):415-421; doi: 10.1055/s-00311271771.

36. Lago C, Casais L, Dominguez E, Sampaio J. The effects of situational variables on distance covered at various speeds in elite soccer. Eur J Sport Sci. 2010;10(2):103109; doi: 10.1080/17461390903273994. 